\title{
CICLAGEM DE NUTRIENTES EM Eucalyptus grandis W. Hill ex Maiden NO NORTE FLUMINENSE ${ }^{1}$
}

\author{
Gláucio de Mello Cunha ${ }^{2}$, Antonio Carlos da Gama-Rodrigues ${ }^{3}$ e Gilmar Santos Costa ${ }^{4}$
}

\begin{abstract}
RESUMO - A ciclagem de nutrientes em povoamentos de eucalipto permite avaliar possíveis alterações decorrentes de técnicas de manejo aplicadas e possibilita inferir sobre a sustentabilidade das plantações. O objetivo deste trabalho foi estudar a ciclagem de nutrientes e o balanço nutricional em povoamentos de Eucalyptus grandis em primeira rotação ( 8 anos de idade) e em sistema de rebrota (1,5 e 5 anos) na região Norte Fluminense, no período de janeiro a dezembro de 2001. Determinaram-se a biomassa e o conteúdo de nutrientes da parte aérea (folha, galho, casca e lenho) e da serapilheira depositada e acumulada. Efetuou-se o balanço de nutrientes no sistema solo-serapilheira-parte aérea. O talhão de 8 anos apresentou maior diâmetro, área basal e volume de tronco. Por conseguinte, a biomassa e o conteúdo de nutrientes nesse componente foram superiores aos de outros talhões. A produção total de serapilheira e o fluxo anual de nutrientes no sistema planta-solo foram maiores no talhão em primeira rotação. $O$ folhedo representou a maior contribuição para a deposição de nutrientes ao solo, e o balanço de nutrientes foi positivo em todos os talhões avaliados. Entretanto, houve baixa produção de biomassa, especialmente no talhão em primeira rotação, apesar de as reservas de nutrientes do solo permitirem maior produtividade.
\end{abstract}

Palavras-chave: Biomassa, rotação e rebrota.

\section{NUTRIENT CYCLING IN A EUCALYPT PLANTATION (Eucalyptus grandis W. Hill ex Maiden) IN NORTHERN RIO DE JANEIRO STATE}

\begin{abstract}
Nutrient cycling in eucalypt stands allows the evaluation of changes due to management and to make inferences about plantation sustainability. The objective of this study was to evaluate the nutrient cycling in eucalypt (Eucalyptus grandis) in Northern Rio de Janeiro State, Brazil. The evaluation was carried out from January to December 2001, in 8-year-old stands and 1,5 and 5 year old coppicing (second rotation). The biomass and nutrient contents of the aerial part (leaf, branch, bark and bolewood), the litterfall and litter were determined. The nutrient balance in the soil-litter-aboveground system was measured. The 8-yearold stand presented the highest DHB (diameter at breast height), section area and tree trunk volume. Consequently the biomass and the nutrient content in this component were higher than in the other stands. Litterfall production was larger in the 8-year-old stand. The nutrient annual flow in the plant-soil system was larger in the 8year-old stand. Leaf litterfall was the component that contributed the most to nutrient deposition on the soil. Nutrient balance was positive in all stands evaluated. Despite the fact that the soil nutrient resources allows for larger productivity, a low biomass production was found, particularly in the 8-year-old stand.
\end{abstract}

Key word: Biomass, coppicing, rotation.

\footnotetext{
${ }^{1}$ Recebido em 07.11.2003 e aceito para publicação em 20.04.2005.

${ }^{2}$ Centro de Ciências Agrárias da Universidade Federal do Espírito Santo (UFES), 29500-000 Alegre-ES.

${ }^{3}$ Laboratório de Solos do Centro de Ciências e Tecnologias Agropecuárias (CCTA) da Universidade Estadual do Norte Fluminense (UENF), 28013-602 Campos dos Goytacazes-RJ. Bolsista da FAPERJ. E-mail: <tonygama@uenf.br>.

${ }^{4}$ Instituto Superior de Ciências Agrárias da FAETEC-RJ. Av. Wilson Batista, s/n. Parque Aldeia, 28070-620 Campos dos Goytacazes-RJ.
} 


\section{INTRODUÇÃO}

No Norte Fluminense, a demanda energética de origem vegetal é muito elevada em decorrência da existência de um grande pólo ceramista. Parte da demanda madeireira dessa região é suprida por plantios de eucalipto localizados no sul do Estado do Espírito Santo. Historicamente, o Norte Fluminense caracteriza-se como grande produtor de cana-de-açúcar, mas, em razão da desaceleração do proálcool, milhares de hectares de terras da região cultivadas com cana-de-açúcar apresentam baixa produtividade ou estão à margem do processo produtivo. Diante desse panorama, a cultura do eucalipto seria uma das atividades que podem, ao mesmo tempo, suprir a demanda energética local e ser uma opção rentável para o uso da terra.

Apesar da carência tecnológica na implantação e condução da cultura, alguns plantios de eucalipto são encontrados na região Norte Fluminense. O conhecimento da qualidade dos sítios implantados é essencial para se diagnosticar o potencial produtivo da cultura na região, de modo a se constituir em uma alternativa rentável, principalmente em áreas ocupadas por cana-de-açúcar de baixa produtividade e em terras sem uso.

A ciclagem de nutrientes em povoamentos de eucalipto permite avaliar possíveis alterações decorrentes de técnicas de manejo aplicadas e possibilita inferir sobre a sustentabilidade das plantações (GAMARODRIGUES e BARROS, 2002). Neste aspecto, as informações a respeito das quantidades de nutrientes encontradas no solo, na biomassa da parte aérea e na serapilheira são importantes para a compreensão da estrutura do ecossistema e para definição de estratégias para manutenção da sustentabilidade do ecossistema florestal (LIMA, 1996; SPANGENBERG et al., 1996).

Em geral, o eucalipto produz serapilheira relativamente pobre em nutrientes, em razão de sua eficiente ciclagem bioquímica (GAMA-RODRIGUES e BARROS, 2002), contribuindo, assim, pouco para a reposição de nutrientes ao solo. A questão da manutenção da produtividade de plantações de eucalipto em regime de curtas rotações está relacionada com a espécie e com as práticas de manejo aplicadas e com a densidade de plantio (LIMA, 1996; LEITE et al., 1998; GUO e SIMS, 1999). Em plantações comerciais, a prática de adubação no plantio tem beneficiado a produtividade dos sítios (BARROS et al., 1990), e o processo de ciclagem de nutrientes pode contribuir para garantir a sustentabilidade de rebrotas e, ou, rotações subseqüentes, em razão de a serapilheira que se acumula no piso florestal suprir parte da demanda de energia e de nutrientes do ecossistema florestal (BARROS et al., 1990; GUO e SIMS, 1999). Nesse sentido, Faria et al. (2002), enfocando o efeito residual do K, atribuíram à ciclagem de nutrientes, por meio dos resíduos florestais da primeira rotação, como reflexo da aplicação de K no plantio e do crescimento de um povoamento de E. grandis em segunda rotação.

O acúmulo e distribuição de nutrientes nos diversos componentes da planta e no solo podem servir de indicadores de diferenças entre os ecossistemas, em especial no que tange à disponibilidade de nutrientes para as plantas. Dessa maneira, no processo de ciclagem, o retorno de nutrientes por meio da queda de folhas e outras estruturas das árvores constitui a via mais importante do ciclo biogeoquímico, e que se torna mais relevante em condições de solos de baixa fertilidade (REIS e BARROS, 1990). Desse modo, mesmo que o teor de nutrientes na serapilheira provenientes de solos mais pobres seja menor que em solos férteis, ele constitui uma das principais fontes de nutrientes do sistema (GAMA-RODRIGUES e BARROS, 2002).

O objetivo deste trabalho foi estudar a ciclagem de nutrientes e o balanço nutricional em povoamentos de Eucalyptus grandis em primeira rotação e em sistema de rebrota na região Norte Fluminense.

\section{MATERIAL E MÉTODOS}

O trabalho foi desenvolvido no município de São Francisco do Itabapoana, Norte Fluminense, em plantio comercial de Eucalyptus grandis W. Hill ex Maiden. Anteriormente ao cultivo de eucalipto, a área era ocupada por cana-de-açúcar. A finalidade desse sítio florestal é a produção de lenha. Possui área total de aproximadamente 100 ha, ocupada com talhões de diversas idades e regimes de rotação. O período de condução do trabalho foi de janeiro a dezembro de 2001, em três talhões de diferentes idades: talhão com idade de 8 anos, em primeira rotação, com espaçamento de 2,0 x 2,5 m; talhão em segunda rotação, em sistema de rebrotas, com idade de 1,5 ano, com espaçamento de 2,5 x 2,5 $\mathrm{m}$; e outro talhão, com rebrota de cinco anos, em espaçamento de 2,0 x 1,5 m. Os talhões encontramse em Argissolo Amarelo Distrófico típico (EMBRAPA, 
1999), que apresenta na camada de $0-20 \mathrm{~cm}$ as seguintes características texturais: talhão de 8 anos, 660, 50 e $290 \mathrm{~g} \mathrm{~kg}^{-1}$ de areia, silte e argila, respectivamente; talhão de 1,5 ano, 690, 40 e $270 \mathrm{~g} \mathrm{~kg}^{-1}$ de areia, silte e argila, respectivamente; e talhão de 5 anos, 650, 50 e $300 \mathrm{~g}$ $\mathrm{kg}^{-1}$ de areia, silte e argila, respectivamente. Os dados climáticos durante o período experimental foram obtidos pela Estação Evapotranspirométrica UENF/PESAGRO, em Campos dos Goytacazes, RJ (Figura 1).

Na coleta da serapilheira, utilizaram-se coletores de $1 \mathrm{~m}^{2}$ construídos com ripas de madeira e tela sombrite. Esses coletores foram instalados a uma altura de 1 $\mathrm{m}$ da superfície do solo. Em duas áreas com dimensões de 20 × 25 m em cada talhão, quatro coletores por área foram dispostos, a fim de se coletar o material proveniente da parte aérea das árvores. O conteúdo dos coletores foi recolhido a cada 30 dias, sendo fracionado em folhedo (folhas com diferentes graus de senescência), galhos menores que $2 \mathrm{~cm}$ de diâmetro, cascas, flores e frutos. O material separado foi secado em estufa a $60^{\circ} \mathrm{C}$. Nas mesmas áreas de coleta, mediu-se o diâmetro à altura do peito (DAP) em todas as árvores, ressaltando-se que, em duas árvores com dimensões iguais às das médias das áreas, foi quantificada a biomassa (folha, galho, casca e lenho). Retiraram-se amostras de material vegetal para secagem em estufa a $60^{\circ} \mathrm{C}$ para determinação da biomassa. Na cubagem do tronco, adotou-se o mesmo procedimento de Gama-Rodrigues e Barros (2002).

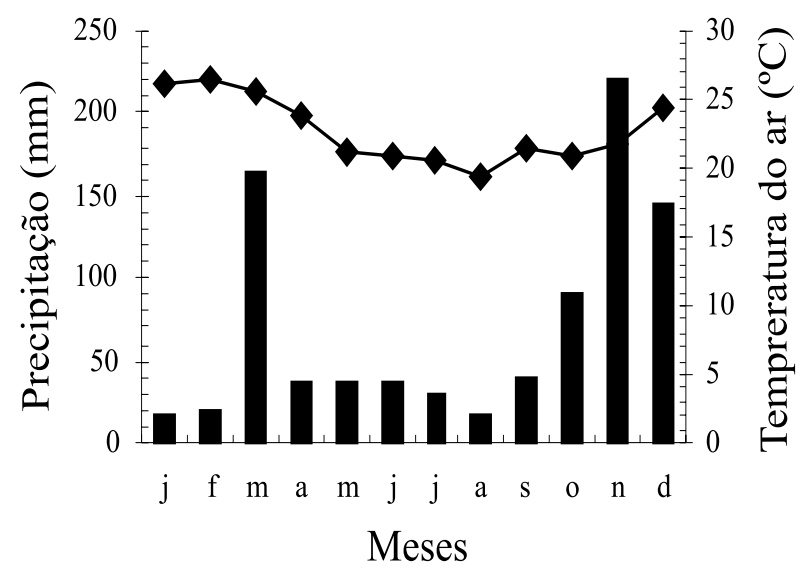

Figura 1-Médias mensais de precipitação pluvial e temperatura média do ar no período de janeiro a dezembro de 2001.

Figure 1-Monthly variation (January-December/2001) rainfall and air temperature in Northern, Rio de Janeiro State.
A amostragem da serapilheira acumulada sobre o solo foi feita duas vezes ao ano, uma no período de inverno e outra no período de verão, utilizandose um gabarito de madeira de dimensões de $0,50 \mathrm{~m}$ x $0,50 \mathrm{~m}$, com quatro repetições por área de $20 \times 25$ $\mathrm{m}$. Nas amostras de tecido vegetal proveniente da serapilheira estocada sobre o solo, da parte aérea das árvores médias dos talhões e da serapilheira mensal, determinaram-se os teores de $\mathrm{P}$ (colorimetricamente, usando como redutor o ácido ascórbico, modificado por Braga e Defelipo, 1974), de K, Ca e Mg (espectrofotometria de absorção atômica), após a digestão nitroperclórica (TEDESCO et al., 1985). O N total foi determinado nas amostras após a digestão sulfúrica, utilizando-se o destilador automático Foss Tecator.

A caracterização da fertilidade do solo foi feita por meio de quatro amostras compostas provenientes de 30 amostras simples, na camada de $0-10 \mathrm{~cm}$. Também foram coletadas amostras de solo, nas camadas de 0 $-20,20-40,40-60$ e $60-80 \mathrm{~cm}$, com oito repetições por parcela, para se determinar o balanço nutricional. Nas amostras de solo determinaram-se o pH em água, $\mathrm{Ca}, \mathrm{Mg}$ e Al trocáveis extraídos em solução $\mathrm{KCl} 1 \mathrm{~mol}$ $\mathrm{L}^{-1}, \mathrm{P}$ e K disponíveis extraídos por Mehlich-1, a acidez potencial extraída com acetato de cálcio $1 \mathrm{~mol} \mathrm{~L}^{-1}$ a pH 7,0 e o carbono orgânico do solo por oxidação com $\mathrm{K}_{2} \mathrm{Cr}_{2} \mathrm{O}_{7}$ 0,4 mol L-1, conforme Defelipo e Ribeiro (1981). A análise granulométrica foi feita segundo a Embrapa (1997). A estimativa do conteúdo de nutrientes nos componentes das árvores foi realizada multiplicandose as concentrações de nutrientes pelos respectivos valores de massa de matéria seca.

Para o balanço nutricional, tomaram-se valores dos conteúdos dos nutrientes no solo (em diferentes camadas), na serapilheira e nos componentes das árvores, para cada cobertura florestal.

A intensidade do processo de ciclagem bioquímica foi avaliada pelas variações nas concentrações de nutrientes entre folhas e folhedo. Já as variações nas concentrações de nutrientes entre o folhedo e a serapilheira foram indicadoras da intensidade de ciclagem biogeoquímica dos nutrientes contidos na serapilheira (REIS e BARROS, 1990; LEITE et al., 1998; GAMARODRIGUES e BARROS, 2002). A constante de decomposição $k$ foi calculada, dividindo-se a quantidade de folhas que caíram anualmente pela quantidade de folhas acumuladas sobre o solo, conforme Reis e Barros (1990).

R. Árvore, Viçosa-MG, v.29, n.3, p.353-363, 2005 
Na análise de variância dos dados de fitomassa e composição química da serapilheira e dos atributos químicos do solo nos talhões das coberturas florestais, adotou-se o delineamento inteiramente casualizado, a exemplo do procedimento empregado por GamaRodrigues e Barros (2002). Para comparar as médias, foi usado o teste de Tukey a 5\%. Cada talhão florestal foi considerado um tratamento de efeito fixo.

\section{RESULTADOS E DISCUSSÃO}

A caracterização da estrutura dos talhões encontrase no Quadro 1. O talhão em primeira rotação apresentou maior DAP, área basal, sobrevivência e volumes dos componentes do fuste. O DAP médio das rebrotas foi igual; no entanto, na rebrota de 5 anos notou-se que a área basal e os volumes de tronco, de lenho e de casca são maiores, mesmo com menor índice de sobrevivência, devido à sua maior densidade de árvores. A estimativa do volume de tronco em árvores em primeira rotação indicou que o $E$. grandis possui rendimento inferior aos sítios menos produtivos de E. grandis avaliados por Santana et al. (1999). A baixa produtividade do primeiro corte provavelmente deveu-se a fatores como: material genético não melhorado, proveniente de sementes da região, tratos culturais inadequados e aproveitamento da fertilidade residual do cultivo da cana-de-açúcar. Contudo, maior produtividade pode ser alcançada com o uso de clones melhorados, tratos culturais adequados (cultivo, controle de formigas e desbrotas), associados ao manejo da fertilidade do solo que atenda às necessidades das plantas.

Os solos dos talhões são ácidos, com baixo nível de fertilidade, diferindo-se apenas quanto ao teor de $\mathrm{K}$, provavelmente pelo efeito residual do cultivo da cana-de-açúcar (Quadro 2). O baixo nível de fertilidade em parte se deve à própria origem dos solos dos tabuleiros costeiros e, também, ao uso intensivo com a cultura da cana-de-açúcar.

A produção de matéria seca total foi maior no talhão de 8 anos (primeira rotação) (Quadro 3); no entanto, essa produção foi bem inferior à encontrada por Santana et al. (1999) em São Paulo e por Morais et al. (1990) em Viçosa, MG. A proporção de folhas em relação ao total de matéria seca da árvore diminuiu com o aumento da idade da planta. Na rebrota de 1,5 ano, em razão da fase inicial de desenvolvimento das árvores, boa parte dos carboidratos é direcionada para a produção de biomassa da copa. Espera-se que com o tempo as copas comecem a competir entre si, condicionando aumento da produção relativa do tronco, com a diminuição gradativa da produção de folhas e ramos (REIS e BARROS, 1990). Dados de Caldeira et al. (2000) indicam proporção da ordem de $22,5 \%$ de matéria seca de folhas em relação à matéria seca total de árvores de Acacia mearnsii de 2,4 anos, evidenciando a maior participação relativa de folhas na biomassa total em povoamentos florestais jovens.

Na rebrota de 1,5 ano, o conteúdo de $\mathrm{N}$ e $\mathrm{P}$ nas folhas foi maior do que na rebrota de 5 anos e com valores iguais, à exceção do $\mathrm{K}$, na primeira rotação (Quadro 4). Esse resultado deveu-se à maior biomassa de folhas da rebrota de 1,5 ano (Quadro 3), bem como à maior concentração de nutrientes, geralmente encontrada em folhas de povoamentos mais jovens. Em E. globulus com 4 anos idade, o componente madeira representou $63 \%$ da biomassa, no entanto as folhas acumularam $60 \%$ de $\mathrm{N}, 41 \%$ de $\mathrm{P}, 32 \%$ de $\mathrm{K}, 25 \%$ de $\mathrm{Ca}$ e $35 \%$ de $\mathrm{Mg}$ do total de nutrientes da árvore (SCHUMACHER, 1998).

Quadro 1 - Diâmetro à altura do peito (DAP), área basal (AB), volume de tronco (VTR), volume do lenho (VL) e volume de casca (VC) em três talhões de Eucalyptus grandis. Os valores entre parênteses indicam o erro-padrão

Table 1 -Diameter at breast height $(D B H)$, section area $(A S)$, tree trunk volume $(T V)$, bolewood volume (BwV), bark volume $(B k V)$ of three Eucalypt stands. Standard-errors in parentheses

\begin{tabular}{|c|c|c|c|c|c|c|c|c|c|}
\hline Talhões ${ }^{1 /}$ & \multicolumn{2}{|c|}{ DAP } & \multicolumn{2}{|c|}{$\mathrm{AB}$} & SOBR & & VTR & VL & $\mathrm{VC}$ \\
\hline & \multicolumn{2}{|c|}{$\mathrm{cm}$} & \multicolumn{2}{|c|}{$\mathrm{m}^{2} / \mathrm{ha}$} & $\%$ & \multicolumn{4}{|c|}{$\mathrm{m} 3 / \mathrm{ha}$} \\
\hline $1^{\circ}$ rotação & $14,33 \mathrm{a}$ & $(4,20)$ & $25,85 \mathrm{a}$ & $(3,87)$ & 74 & $175,01 \mathrm{a}$ & a $(18,37)$ & 148,20 a $(15,39)$ & 26,82 a $(2,98)$ \\
\hline Rebrota 1,5 & $7,68 \mathrm{~b}$ & $(2,26)$ & $7,28 \mathrm{c}$ & $(2,32)$ & 45 & $44,94 \mathrm{c}$ & c $(15,44)$ & $34,50 \mathrm{c}(14,46)$ & $10,44 \mathrm{~b} \quad(0,98)$ \\
\hline Rebrota 5 & $7,68 \mathrm{~b}$ & $(2,49)$ & $11,25 \mathrm{~b}$ & $(1,94)$ & 40 & $106,17 \mathrm{~b}$ & b $(13,26)$ & 81,92 b $\quad(4,43)$ & 24,25 a $(8,82)$ \\
\hline
\end{tabular}

${ }^{1 /} 1^{\circ}$ rotação = eucalipto de 8 anos, rebrota de 1,5 ano e rebrota de 5 anos. Médias seguidas de letras iguais na coluna, para cada característica, não diferem, estatisticamente, pelo teste de Tukey $(\mathrm{p}<0,05)$.

R. Árvore, Viçosa-MG, v.29, n.3, p.353-363, 2005

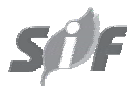


Quadro 2 - Médias de valores de pH e de teores de cálcio, magnésio, alumínio e potássio trocáveis, acidez potencial, fósforo disponível e matéria orgânica (MO) de amostras de solo em três talhões de Eucalyptus grandis. Os valores entre parênteses correspondem ao erro-padrão

Table 2 - Average of $\mathrm{pH}$, content of exchangeable cations (Ca, $\mathrm{Mg}, \mathrm{Al}, \mathrm{H}+\mathrm{Al}, \mathrm{K}), \mathrm{P}$ and organic matter (OM.) of soil samples of three Eucalyptus grandis stands. Standard errors in parentheses

\begin{tabular}{|c|c|c|c|c|c|c|c|c|}
\hline \multirow{3}{*}{$\begin{array}{l}\text { Talhões }^{1 /} \\
1^{\circ} \text { rotacão }\end{array}$} & \multicolumn{2}{|c|}{$\mathrm{pH}$} & \multicolumn{2}{|c|}{$\mathrm{Ca}$} & \multicolumn{2}{|c|}{$\mathrm{Mg}$} & \multicolumn{2}{|c|}{$\mathrm{Al}$} \\
\hline & \multicolumn{2}{|c|}{$\mathrm{H}_{2} \mathrm{O}$} & \multicolumn{6}{|c|}{$\mathrm{cmol}_{\mathrm{c}(+)} \mathrm{kg}^{-1}$} \\
\hline & 4,88 & $(0,21)$ & $0,63 \mathrm{a}$ & $(0,11)$ & $0,32 \mathrm{a}$ & $(0,09)$ & $0,45 \mathrm{a}$ & $(0,12)$ \\
\hline Rebrota 1,5 & 4,82 & $(0,32)$ & $0,73 \mathrm{a}$ & $(0,23)$ & $0,29 \mathrm{a}$ & $(0,06)$ & $0,36 \mathrm{a}$ & $(0,09)$ \\
\hline \multirow[t]{2}{*}{ Rebrota 5} & 4,89 & $(0,19)$ & $0,62 \mathrm{a}$ & $(0,15)$ & $0,35 \mathrm{a}$ & $(0,04)$ & $0,36 \mathrm{a}$ & $(0,07)$ \\
\hline & \multicolumn{2}{|c|}{$\begin{array}{c}\mathrm{H}+\mathrm{Al} \\
\mathrm{cmol}_{\mathrm{c}(+)} \mathrm{kg}^{-1}\end{array}$} & \multicolumn{2}{|c|}{$\bar{K}$} & \multicolumn{2}{|c|}{$\bar{P}$} & \multicolumn{2}{|c|}{$\begin{array}{l}\text { M.O. } \\
\mathrm{g} \mathrm{kg}^{-1}\end{array}$} \\
\hline $1^{\circ}$ rotação & 3,80 & $(1,25)$ & 34,75 a & $(7,45)$ & 3,08 a & $(2,13)$ & $22,1 \mathrm{a}$ & $(5,48)$ \\
\hline Rebrota 1,5 & 4,29 & $(1,39)$ & $22,50 \mathrm{~b}$ & $(6,39)$ & $2,82 \mathrm{a}$ & $(1,98)$ & 19,6 a & $(3,29)$ \\
\hline Rebrota 5 & 3,62 & $(1,18)$ & $25,00 \mathrm{~b}$ & $(4,75)$ & $2,72 \mathrm{a}$ & $(1,44)$ & $20,9 \mathrm{a}$ & $(4,13)$ \\
\hline
\end{tabular}

${ }^{1 /} 1^{\circ}$ rotação = eucalipto de 8 anos, rebrota de 1,5 ano e rebrota de 5 anos. Médias seguidas de letras iguais na coluna, para cada característica, não diferem, estatisticamente, pelo teste de Tukey $(\mathrm{p}<0,05)$.

Quadro 3 - Produção de matéria seca total e dos componentes de árvores de Eucalyptus grandis, em três idades, no Norte Fluminense

Table 3 - Production of total dry matter and tree components of three Eucalyptus grandis stands

\begin{tabular}{|c|c|c|c|c|c|c|}
\hline \multirow[t]{3}{*}{ Compor } & \multicolumn{6}{|c|}{ Talhões $^{1 /}$} \\
\hline & \multicolumn{2}{|c|}{$1^{\circ}$ rotação } & \multicolumn{2}{|c|}{ Rebrota 1,5} & \multicolumn{2}{|c|}{ Rebrota 5} \\
\hline & $\mathrm{Kg} \mathrm{ha}^{-1}$ & $\%$ & $\mathrm{~kg} \mathrm{ha}^{-1}$ & $\%$ & $\mathrm{~kg} \mathrm{ha}^{-1}$ & $\%$ \\
\hline$\overline{\text { Folha }}$ & $2.770 \mathrm{a}$ & 2,4 & $2.600 \mathrm{a}$ & 9,3 & $2.170 \mathrm{~b}$ & 3,5 \\
\hline Galho & $11.170 \mathrm{a}$ & 9,6 & $2.920 \mathrm{c}$ & 10,4 & $6.510 \mathrm{~b}$ & 10,6 \\
\hline Casca & $12.910 \mathrm{a}$ & 11,1 & $4.200 \mathrm{c}$ & 15,0 & $9.170 \mathrm{~b}$ & 15,0 \\
\hline Lenho & $88.370 \mathrm{a}$ & 76,9 & $18.290 \mathrm{c}$ & 65,3 & $43.330 \mathrm{~b}$ & 70,9 \\
\hline Total & $115.820 \mathrm{a}$ & 100,0 & $28.010 \mathrm{c}$ & 100,0 & $61.180 \mathrm{~b}$ & 100,0 \\
\hline
\end{tabular}

${ }^{1 /} 1^{\circ}$ rotação $=$ eucalipto de 8 anos rebrota de 1,5 ano e rebrota de 5 anos. Médias seguidas de letras iguais na linha, para cada característica, não diferem, estatisticamente, pelo teste de Tukey $(\mathrm{p}<0,05)$.

Nos três talhões avaliados, a sazonalidade da queda de folhas foi muito semelhante durante o período experimental (Figura 2). A maior produção de serapilheira ocorreu em dezembro, especialmente no talhão em primeira rotação. A produção de galho apresentou tendência muito similar nos três talhões, mas com produção ligeiramente superior na rebrota de 5 anos, no período de outubro a fevereiro. A produção de casca foi concentrada no período de agosto a novembro. As estruturas reprodutivas foram encontradas em maior quantidade no talhão em primeira rotação, em razão da maturidade das plantas. Diversos trabalhos constataram maior queda de folhedo no período de verão (GUO e SIMS, 1999; GAMA-RODRIGUES e BARROS, 2002). No sudeste da Bahia, em solos também dos tabuleiros costeiros, Gama-Rodrigues e Barros (2002) encontraram a mesma tendência em plantio misto de E. grandis e E. urophylla em produzir maiores quantidades de serapilheira no período de maior precipitação pluviométrica. Isso indica a forte influência dos fatores climáticos na sazonalidade da produção de serapilheira de ecossistemas florestais (LUIZ̃̃O e SCHUBART, 1987; SCHUMACHER et al., 1994; LISANEWORK e MICHELSEN, 1994). Dentre os fatores climáticos, têm sido atribuído à temperatura os efeitos relacionados com o aumento da senescência de folhas no verão (ATTIWILL e ADAMS, 1978).

O aporte de serapilheira foi maior nos talhões em primeira rotação e na rebrota de 5 anos (Quadro 5). A maior contribuição foi do folhedo com 62, 76 e 55\% nos talhões em primeira rotação e nas rebrotas de 1,5 e 5 anos, respectivamente. Notou-se que a produção total de serapilheira cresceu em razão da idade dos talhões. Estudos com E. diversicolor no sudoeste da Austrália evidenciaram que a estabilidade da produção total de serapilheira ocorreu a partir do oitavo ano (O’CONNEL, 1989). 
Quadro 4 - Conteúdo e distribuição relativa de nutrientes dos componentes das árvores de Eucalyptus grandis em três idades. Os valores entre parênteses correspondem ao erro-padrão

Table 4 - Nutrient content and relative distribution of tree components of Eucalyptus grandis. Standard errors in parentheses

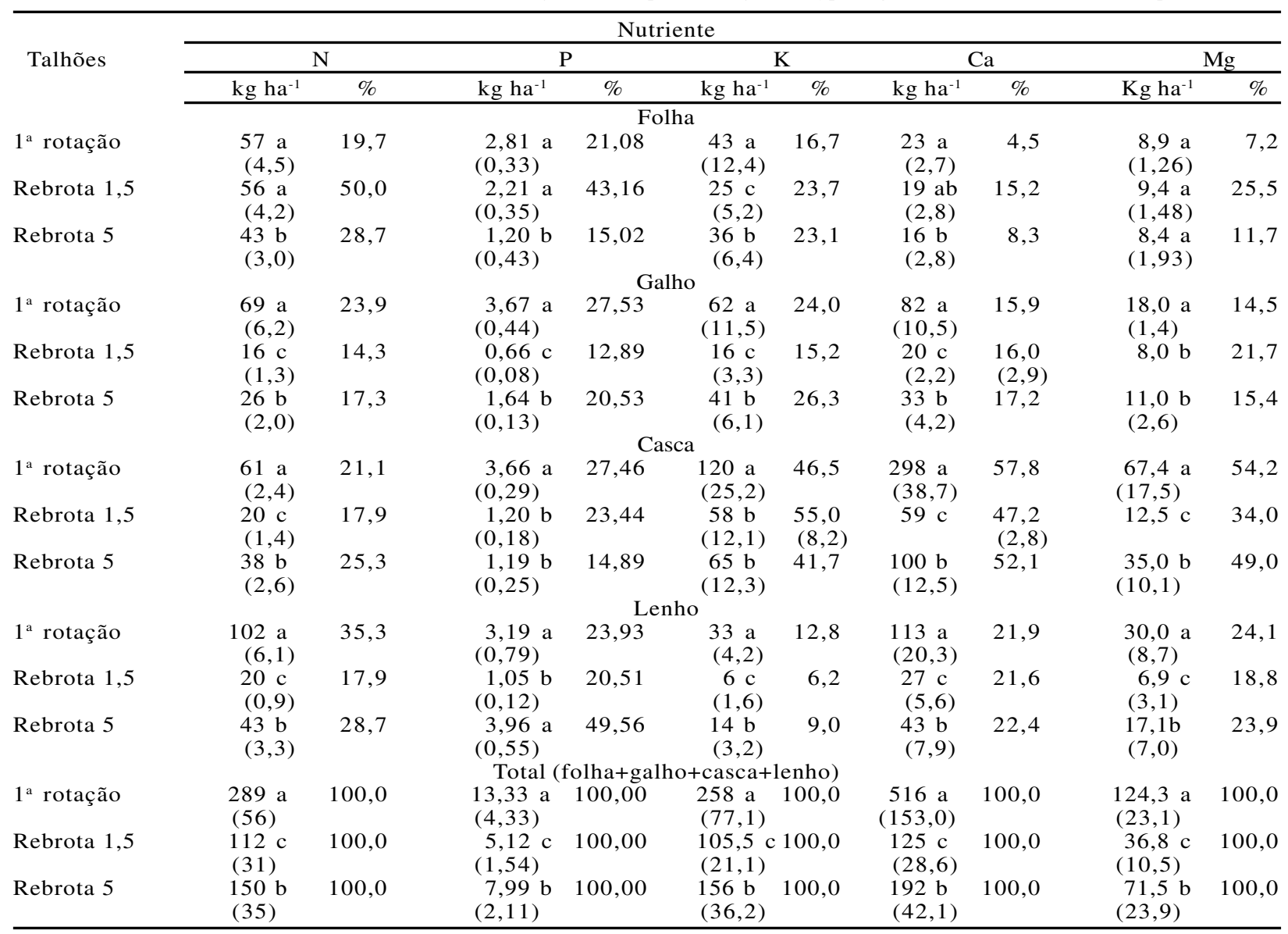

Médias seguidas de letras iguais na coluna, para cada característica, não diferem, estatisticamente, pelo teste de Tukey ( $p<0,05)$.
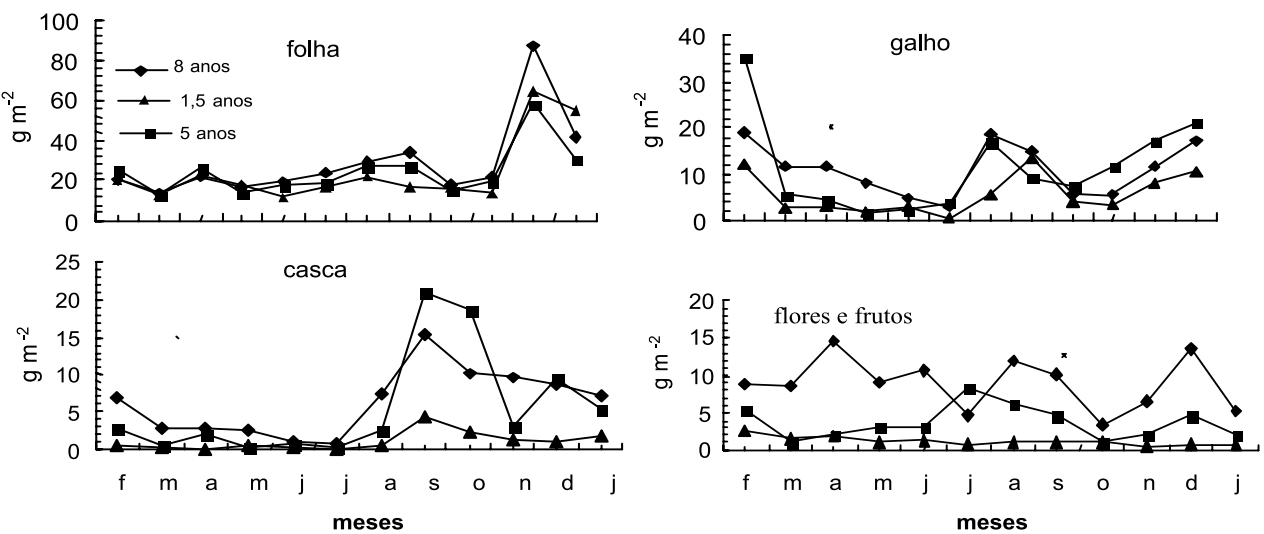

Figura 2 - Sazonalidade da produção de componentes da serapilheira em três talhões de Eucalyptus grandis em três idades, no Norte Fluminense.

Figure 2 - Monthly variation (2001) of leaf litterfall, branch, bark, flower and fruits in three Eucalyptus grandis stands in Northern Rio de Janeiro State.

R. Árvore, Viçosa-MG, v.29, n.3, p.353-363, 2005 
Quadro 5 - Produção total de serapilheira em três talhões de Eucalyptus grandis, no período de janeiro a dezembro de 2001. Os valores entre parênteses correspondem ao erro-padrão

Table 5 - Annual Litterfall in three Eucalyptus grandis stands, from January to December 2001. Standard errors in parentheses

\begin{tabular}{lccccc}
\hline Talhões $^{1 /}$ & Folhedo & Galho & Casca & $\begin{array}{c}\text { Estrutura } \\
\text { Reprodutiva }\end{array}$ \\
\hline \multirow{5}{*}{$1^{\text {a }}$ rotação } & $3,48 \mathrm{a}$ & $1,31 \mathrm{a}$ & $0,74 \mathrm{a}$ & $0,10 \mathrm{~b}$ & $5,63 \mathrm{a}$ \\
& $(0,75)$ & $(0,61)$ & $(0,41)$ & $(0,07)$ & $(1,84)$ \\
Rebrota 1,5 & $2,90 \mathrm{~b}$ & $0,67 \mathrm{~b}$ & $0,12 \mathrm{~b}$ & $0,13 \mathrm{~b}$ & $3,82 \mathrm{~b}$ \\
& $(0,45)$ & $(0,51)$ & $(0,04)$ & $(0,07)$ & $(1,00)$ \\
Rebrota 5 & $2,92 \mathrm{~b}$ & $1,35 \mathrm{a}$ & $0,66 \mathrm{a}$ & $0,43 \mathrm{a}$ & $5,36 \mathrm{a}$ \\
& $(0,68)$ & $(0,64)$ & 0,49 & $(0,25)$ & $(2,09)$ \\
\hline
\end{tabular}

${ }^{1 /} 1^{\circ}$ rotação $=$ eucalipto de 8 anos rebrota de 1,5 ano e rebrota de 5 anos. Médias seguidas de letras iguais na coluna, para cada característica, não diferem, estatisticamente, pelo teste de Tukey $(\mathrm{p}<0,05)$.

Os dados referentes à concentração média anual de nutrientes nos componentes da serapilheira produzida são apresentados no Quadro 6. Houve tendência de as concentrações sofrerem pouca alteração em função da idade, principalmente no folhedo. No galho e na casca ocorreu pequena variação nos teores de Ca e $\mathrm{K}$ conforme a idade. Desse modo, a diferença de aporte de nutrientes entre as idades é decorrente, em grande parte, da variação de massa dos diversos componentes da serapilheira que cai ao longo do ano (Quadro 7). $\mathrm{O}$ fluxo anual de $\mathrm{N}$ e Ca no sistema planta-solo foi maior no talhão em primeira rotação, seguido das rebrotas de 5 anos e 1,5 ano. O folhedo foi o componente que mais contribuiu para a deposição de nutrientes no solo, de maneira que a manutenção da fertilidade do solo em plantios de eucalipto, principalmente nas idades mais jovens, é diretamente dependente da quantidade e qualidade do folhedo e, por conseguinte, da sua taxa de decomposição. Em solos de baixa fertilidade natural, a deposição de folhedo torna-se a principal fonte de nutrientes para o desenvolvimento da planta. Em plantações de eucalipto, o retorno ao solo de $\mathrm{N}$ pode variar entre $18-140 \mathrm{~kg} \mathrm{ha}^{-1} \mathrm{ano}^{-1}$ e de P entre $0,9 \mathrm{e}$ $8 \mathrm{~kg} \mathrm{ha}^{-1} \mathrm{ano}^{-1}$ (REIS e BARROS, 1990; ATTIWILL e ADAMS, 1993; GUO e SIMS, 1999).

O estoque de serapilheira sobre o solo variou entre os talhões (Quadro 8). Em geral, o estoque de serapilheira é função da biomassa do povoamento. No entanto, a rebrota de 1,5 ano apresentou maior estoque de serapilheira do que o talhão em primeira rotação. Isso seria decorrência do ciclo anterior, incluindo galhos e folhas que não foram retirados das áreas após a colheita. Na rebrota de 5 anos, a biomassa da serapilheira acumulada foi maior do que nos outros talhões, e as razões para isso foram: o resíduo do manejo das brotações (que

Quadro 6 - Concentração média anual de nutrientes na serapilheira produzida de Eucalyptus grandis em três idades, no Norte Fluminense. Os valores entre parênteses correspondem ao erro-padrão

Table 6 - Nutrient concentration in the litterfall of three Eucalyptus grandis stands in Northern Rio de Janeiro. Standard errors in parentheses

\begin{tabular}{|c|c|c|c|c|c|}
\hline \multirow{3}{*}{ Talhões } & $\mathrm{N}$ & $\mathrm{P}$ & $\mathrm{K}$ & $\mathrm{Ca}$ & $\mathrm{Mg}$ \\
\hline & \multicolumn{5}{|c|}{$\mathrm{g} \mathrm{kg}^{-1}$} \\
\hline & \multicolumn{5}{|c|}{ Folhedo } \\
\hline $1^{\mathrm{a}}$ rotação & 11,04 a $(0,95)$ & 0,3 a $(0,02)$ & 3,56 a $(0,48)$ & 8,46 b $(0,41)$ & 2,53 a $(0,21)$ \\
\hline Rebrota 1,5 & 10,47 b $(1,03)$ & 0,3 a $(0,01)$ & 3,40 a $(0,63)$ & 8,73 b $(0,33)$ & 2,28 a $(0,13)$ \\
\hline Rebrota 5 & \multicolumn{5}{|c|}{ Galho } \\
\hline $1^{\mathrm{a}}$ rotação & 5,21 a $(0,61)$ & 0,1 a $(0,03)$ & $2,49 \mathrm{~b}(0,63)$ & $7,57 \mathrm{c}(0,61)$ & 1,79 a $(0,19)$ \\
\hline Rebrota 1,5 & 4,93 a $(0,92)$ & 0,1 a $(0,04)$ & $2,67 \mathrm{~b}(0,55)$ & 9,92 a $(0,64)$ & 1,57 a $(0,23)$ \\
\hline Rebrota 5 & \multicolumn{5}{|c|}{ Casca } \\
\hline $1^{\mathrm{a}}$ rotação & 5,58 a $(0,61)$ & 0,2 a $(0,01)$ & $1,17 \mathrm{~b}(0,31)$ & 4,82 a $(0,51)$ & 1,48 a $(0,25)$ \\
\hline Rebrota 1,5 & 5,39 a $(0,77)$ & 0,1 a $(0,05)$ & 2,35 a $(0,22)$ & 3,93 b $(0,45)$ & 1,06 a $(0,35)$ \\
\hline Rebrota 5 & \multicolumn{5}{|c|}{ Estruturas reprodutivas } \\
\hline $1^{\mathrm{a}}$ rotação & $6,15 \mathrm{~b}(0,55)$ & 0,3 a $(0,03)$ & 8,06 b $(0,96)$ & 6,13 a $(0,53)$ & 2,34 a $(0,48)$ \\
\hline Rebrota 1,5 & $6,48 \mathrm{ab}(0,73)$ & 0,3 a $(0,01)$ & 8,13 b $(0,99)$ & 5,27 b $(0,51)$ & 1,65 b $(0,39)$ \\
\hline Rebrota 5 & 6,75 a $(0,41)$ & 0,3 a $(0,04)$ & 9,45 a $(0,81)$ & 6,21 a $(0,44)$ & 2,55 a $(0,36)$ \\
\hline
\end{tabular}

Médias seguidas de letras iguais na coluna, para cada característica, não diferem, estatisticamente, pelo teste de Tukey (p <0,05). 
consistiu em deixar de dois a três ramos em crescimento), em parte os resíduos do ciclo anterior (galhos de maior diâmetro e pontas das árvores) e, ainda, o próprio efeito da deposição da serapilheira. A biomassa foliar da serapilheira correspondeu a 39, 31 e $36 \%$ dos estoques totais nos talhões de 8 anos, rebrotas de 1,5 ano e de 5 anos, respectivamente. Assim, o sistema de rebrota praticamente não alterou a proporção das folhas (em que a dinâmica de decomposição é mais intensa do que o galho e a casca) na biomassa total da serapilheira estocada. As diferenças encontradas no estoque entre os talhões devem, em sua maior parte, às estruturas mais resistentes à decomposição, como galhos e troncos de pequeno diâmetro, que possuem teores mais elevados de lignina (SWIFT et al., 1979).

A constante de decomposição $k$ da serapilheira foliar para o talhão em primeira rotação foi de 0,93. Nas rebrotas de 1,5 e 5 anos, os valores de $k$ foram de 0,67 e 0,35 , respectivamente. O eucalipto no sistema de rebrota preserva algum resíduo do ciclo anterior, havendo ainda o manejo das brotações. Desse modo, a estimativa da constante $k$ da decomposição deve ser interpretada com certas limitações, em razão de a taxa de adição anual de material não ser constante (BIRK e SIMPSON, 1980).

Quadro 7 - Fluxo de nutrientes pela queda de serapilheira em três idades de Eucalyptus grandis. Os valores entre parênteses representam o porcentual em relação ao total ${ }^{1}$ e ao erro-padrão ${ }^{2}$

Table 7 - Annual rate nutrients in litterfall of three Eucalyptus grandis stands in Northern Rio de Janeiro. Percent from the total $^{1}$ and the standard-errors ${ }^{2}$ in parentheses

\begin{tabular}{|c|c|c|c|c|c|c|c|c|c|c|}
\hline \multirow[t]{3}{*}{ Talhão } & \multirow{2}{*}{\multicolumn{2}{|c|}{$\mathrm{N}$}} & \multicolumn{2}{|c|}{$\mathrm{P}$} & \multicolumn{2}{|c|}{$\mathrm{K}$} & \multicolumn{2}{|c|}{$\mathrm{Ca}$} & \multicolumn{2}{|c|}{$\mathrm{Mg}$} \\
\hline & & & \multicolumn{8}{|c|}{$\mathrm{kg} \mathrm{ha}^{-1}$} \\
\hline & & & & & Fol & & & & & \\
\hline $1^{\text {a }}$ rotação & $\begin{array}{c}38,49 \text { a } \\
(3,2)^{2}\end{array}$ & $(68)^{1}$ & $\begin{array}{l}0,97 \mathrm{a} \\
(0,06)\end{array}$ & (63) & $\begin{array}{c}10,45 \mathrm{~b} \\
(1,43)\end{array}$ & (45) & $\begin{array}{c}30,21 \text { a } \\
(5,2)\end{array}$ & $(61)$ & $\begin{array}{c}8,73 \mathrm{a} \\
(0,9)\end{array}$ & $(60)$ \\
\hline Rebrota 1,5 & $\begin{array}{c}30,41 \mathrm{~b} \\
(4,3)\end{array}$ & $(86)$ & $\begin{array}{l}0,90 \mathrm{a} \\
(0,07)\end{array}$ & (88) & $\begin{array}{c}25,97 \mathrm{a} \\
(3,5)\end{array}$ & (78) & $\begin{array}{c}8,50 \mathrm{~b} \\
(1,2)\end{array}$ & $(76)$ & $\begin{array}{c}6,64 \mathrm{a} \\
(1,1)\end{array}$ & (84) \\
\hline \multirow[t]{2}{*}{ Rebrota 5} & $\begin{array}{c}29,97 \mathrm{~b} \\
(2,4)\end{array}$ & $(70)$ & $\begin{array}{l}0,99 \mathrm{a} \\
(0,09)\end{array}$ & $(73)$ & $\begin{array}{c}27,21 \text { a } \\
(3,7)\end{array}$ & $(62)$ & $\begin{array}{c}9,26 \mathrm{~b} \\
(1,7)\end{array}$ & $(49)$ & $\begin{array}{c}8,17 \text { a } \\
(1,2)\end{array}$ & $(65)$ \\
\hline & \multicolumn{10}{|c|}{ Galho } \\
\hline $1^{\text {a }}$ rotação & $\begin{array}{c}6,81 \text { a } \\
(1,8)\end{array}$ & $(12)$ & $\begin{array}{l}0,15 \text { a } \\
(0,01)\end{array}$ & (10) & $\begin{array}{c}3,13 \mathrm{c} \\
(0,5)\end{array}$ & (14) & $\begin{array}{c}9,96 \mathrm{a} \\
(1,7)\end{array}$ & (20) & $\begin{array}{c}2,26 \mathrm{a} \\
(0,6)\end{array}$ & $(15)$ \\
\hline Rebrota 1,5 & $\begin{array}{c}3,30 \mathrm{~b} \\
(1,4)\end{array}$ & (9) & $\begin{array}{l}0,07 \mathrm{~b} \\
(0,01)\end{array}$ & (7) & $\begin{array}{c}6,29 \mathrm{~b} \\
(0,6)\end{array}$ & (19) & $\begin{array}{c}1,60 \mathrm{c} \\
(0,4)\end{array}$ & $(14)$ & $\begin{array}{c}0,93 \mathrm{~b} \\
(0,1)\end{array}$ & (12) \\
\hline \multirow[t]{2}{*}{ Rebrota 5} & $\begin{array}{c}6,64 \mathrm{a} \\
(1,3)\end{array}$ & $(15)$ & $\begin{array}{l}0,19 \mathrm{a} \\
(0,01)\end{array}$ & (14) & $\begin{array}{c}11,62 \mathrm{a} \\
(1,2)\end{array}$ & (26) & $\begin{array}{c}4,83 \mathrm{~b} \\
(0,9)\end{array}$ & $(26)$ & $\begin{array}{c}2,33 \mathrm{a} \\
(0,9)\end{array}$ & (18) \\
\hline & \multicolumn{10}{|c|}{ Casca } \\
\hline $1^{\text {a }}$ rotação & $\begin{array}{c}4,16 \mathrm{a} \\
(1,2)\end{array}$ & (7) & $\begin{array}{l}0,11 \mathrm{a} \\
(0,01)\end{array}$ & (7) & $\begin{array}{c}0,75 \mathrm{~b} \\
(0.2)\end{array}$ & (3) & $\begin{array}{c}2,67 \mathrm{a} \\
(1.0)\end{array}$ & (5) & $\begin{array}{l}1,01 \mathrm{a} \\
(0,51)\end{array}$ & (7) \\
\hline Rebrota 1,5 & $\begin{array}{c}0,67 \mathrm{~b} \\
(0,4)\end{array}$ & (2) & $\begin{array}{c}0,01 \mathrm{~b} \\
(0,002)\end{array}$ & (1) & $\begin{array}{c}0,34 \mathrm{~b} \\
(0,2)\end{array}$ & (1) & $\begin{array}{l}0,09 \mathrm{c} \\
(0,05)\end{array}$ & (1) & $\begin{array}{l}0,12 \mathrm{~b} \\
(0,05)\end{array}$ & (2) \\
\hline \multirow[t]{2}{*}{ Rebrota 5} & $\begin{array}{c}3,39 a \\
(0,7)\end{array}$ & (8) & $\begin{array}{c}0,06 \mathrm{ab} \\
(0,01)\end{array}$ & (4) & $\begin{array}{c}2,72 \mathrm{a} \\
(0,9)\end{array}$ & (6) & $\begin{array}{l}0,52 \mathrm{~b} \\
(0,21)\end{array}$ & (3) & $\begin{array}{l}0,96 \mathrm{a} \\
(0,55)\end{array}$ & (8) \\
\hline & \multicolumn{10}{|c|}{ Estruturas reprodutivas } \\
\hline $1^{\text {a }}$ rotação & $\begin{array}{c}6,92 \text { a } \\
(2,3)\end{array}$ & (12) & $\begin{array}{l}0,31 \mathrm{a} \\
(0,15)\end{array}$ & (20) & $\begin{array}{c}8,71 \mathrm{a} \\
(3,9)\end{array}$ & $(38)$ & $\begin{array}{c}6,96 \text { a } \\
(4,2)\end{array}$ & (14) & $\begin{array}{c}2,59 \mathrm{a} \\
(1,2)\end{array}$ & (18) \\
\hline Rebrota 1,5 & $\begin{array}{c}0,91 \mathrm{c} \\
(0,7)\end{array}$ & (3) & $\begin{array}{l}0,04 \mathrm{c} \\
(0,02)\end{array}$ & (4) & $\begin{array}{c}0,71 \mathrm{c} \\
(0,4)\end{array}$ & (2) & $\begin{array}{c}1,04 \mathrm{c} \\
(0,6)\end{array}$ & (9) & $\begin{array}{c}0,24 \mathrm{c} \\
(0,1)\end{array}$ & (3) \\
\hline \multirow[t]{2}{*}{ Rebrota 5} & $\begin{array}{c}2,96 \mathrm{~b} \\
(1,6)\end{array}$ & (7) & $\begin{array}{l}0,11 \mathrm{~b} \\
(0,06)\end{array}$ & (8) & $\begin{array}{c}2,61 \mathrm{~b} \\
(1,5)\end{array}$ & (6) & $\begin{array}{c}4,21 \mathrm{~b} \\
(1,9)\end{array}$ & $(22)$ & $\begin{array}{c}1,14 \mathrm{~b} \\
(0,6)\end{array}$ & (9) \\
\hline & \multicolumn{10}{|c|}{ Total (folha+galho+casca+lenho) } \\
\hline $1^{\text {a }}$ rotação & $\begin{array}{c}56,38 \text { a } \\
(10,2)\end{array}$ & $(100)$ & $\begin{array}{l}1,54 \mathrm{a} \\
(0,32)\end{array}$ & $(100)$ & $\begin{array}{c}23,04 \mathrm{c} \\
(6,5)\end{array}$ & $(100)$ & $\begin{array}{c}49,80 \text { a } \\
(12,5)\end{array}$ & $(100)$ & $\begin{array}{c}14,59 \mathrm{a} \\
(1,4)\end{array}$ & $(100)$ \\
\hline Rebrota 1,5 & $\begin{array}{c}35,29 \mathrm{c} \\
(3,2)\end{array}$ & $(100)$ & $\begin{array}{l}1,02 \mathrm{~b} \\
(0,16)\end{array}$ & $(100)$ & $\begin{array}{c}33,31 \mathrm{~b} \\
(3,2)\end{array}$ & $(100)$ & $\begin{array}{c}11,23 \mathrm{~b} \\
(2,1)\end{array}$ & $(100)$ & $\begin{array}{c}7,93 \mathrm{~b} \\
(1,1)\end{array}$ & $(100)$ \\
\hline Rebrota 5 & $\begin{array}{c}42,96 \mathrm{~b} \\
(5,8)\end{array}$ & $(100)$ & $\begin{array}{c}1,35 \mathrm{ab} \\
(0,19)\end{array}$ & $(100)$ & $\begin{array}{c}44,16 \text { a } \\
(7,5)\end{array}$ & $(100)$ & $\begin{array}{c}18,82 \mathrm{~b} \\
(4,1)\end{array}$ & $(100)$ & $\begin{array}{c}12,60 \mathrm{a} \\
(2,5)\end{array}$ & $(100)$ \\
\hline
\end{tabular}

Médias seguidas de letras iguais na coluna, para cada característica, não diferem, estatisticamente, pelo teste de Tukey (p<0,05).

R. Árvore, Viçosa-MG, v.29, n.3, p.353-363, 2005 
Quadro 8 - Biomassa total e foliar da serapilheira acumulada sobre o solo e concentração de nutrientes da serapilheira foliar em Eucalyptus grandis em três idades, no Norte Fluminense. Os valores entre parênteses indicam o erro-padrão

Table 8 - Total and leaf litter biomass and leaf litter nutrient concentration of three Eucalyptus grandis stands. Standard errors in parentheses

\begin{tabular}{|c|c|c|c|c|c|c|c|}
\hline Talhões & Serapilheira & Folha & $\mathrm{N}$ & $\mathrm{P}$ & $\mathrm{K}$ & $\mathrm{Ca}$ & $\mathrm{Mg}$ \\
\hline & \multicolumn{2}{|c|}{$\mathrm{Mg} \mathrm{ha}^{-1}$} & \multicolumn{5}{|c|}{$\mathrm{g} \mathrm{kg}^{-1}$} \\
\hline $1^{a}$ rotação & $\begin{array}{c}9,56 \mathrm{c} \\
(1,85)\end{array}$ & $\begin{array}{c}3,74 \mathrm{c} \\
(0,85)\end{array}$ & 10,04 & 0,33 & 0,83 & 10,97 & 2,02 \\
\hline Rebrota 1,5 & $\begin{array}{l}13,85 \mathrm{~b} \\
(3,25)\end{array}$ & $\begin{array}{l}4,33 \mathrm{~b} \\
(0,93)\end{array}$ & 10,74 & 0,30 & 0,75 & 12,02 & 2,07 \\
\hline Rebrota 5 & $\begin{array}{l}22,97 \text { a } \\
(6,29)\end{array}$ & $\begin{array}{l}8,31 \text { a } \\
(1,54)\end{array}$ & 12,00 & 0,31 & 0,87 & 10,21 & 2,10 \\
\hline
\end{tabular}

Médias seguidas de letras iguais na coluna, para cada característica, não diferem, estatisticamente, pelo teste de Tukey (p < 0,05).

O nutriente mais intensamente retranslocado (ciclagem bioquímica) foi o K. A retranslocação de N e $\mathrm{P}$ foi maior nos talhões em primeira rotação e rebrota de 5 anos. Para o Mg não houve distinção entre os talhões. Entretanto, em razão de o Ca ser praticamente imóvel no floema, não se observou variação negativa entre a concentração do folhedo e da folha antes da senescência (Quadro 9). A taxa de ciclagem bioquímica depende da idade do sítio florestal (REIS e BARROS, 1990). Em E. grandis com idade de 51 meses, a taxa de retranslocação de $\mathrm{P}$ foi da ordem de 10,8\%; aos 60 meses de idade, a taxa de retranslocação de $P$ aumentou para 34,2\% (FERREIRA, 1984, citado por REIS e BARROS, 1990). Desse modo, na colheita de madeira de árvores em idades mais jovens, a imobilização de nutrientes seria menor. Assim, em sistema de corte raso, na idade mais avançada poderia ocorrer maior exaustão relativa de nutrientes do solo.

Houve intensa taxa de mineralização de K nos três talhões - indicado pela ciclagem biogeoquímica (valores negativos). Essa dinâmica também foi observada, em menor magnitude, para o Mg e o P. No entanto, o N nas duas rebrotas e o Ca nos três talhões os valores positivos apresentados indicam a imobilização desses nutrientes na serapilheira (Quadro 9).

O balanço de nutrientes foi positivo em todos os talhões avaliados, evidenciando-se maior reserva de nutrientes no solo do que absorvido pelas plantas. No entanto, a disponibilidade de $\mathrm{P}$ no solo, no talhão em primeira rotação é muito similar ao total de $\mathrm{P}$ absorvido. Assim, o P disponível para o crescimento de um novo ciclo de rebrotas nesse talhão depende de reposição de $\mathrm{P}$ ao solo para que não ocorra exaustão das reservas e, conseqüentemente, deficiência de $\mathrm{P}$ nas plantas (Quadro 10). A disponibilidade de nutrientes no solo dos talhões indica possibilidade de maiores produtividades. Assim, a baixa produção de biomassa, especialmente no talhão em primeira rotação (Quadro 3 ), pode ser devida a outros fatores. Do ponto de vista de plantios comerciais mais homogêneos, poder-seia conjeturar a respeito da variabilidade genética das plantas, por se tratar de material oriundo de sementes, ou mesmo o déficit hídrico da região Norte Fluminense (Figura 1), como fatores condicionantes da baixa produtividade do eucalipto neste estudo.

Quadro 9 - Variação porcentual da concentração de nutrientes entre folhedo (Fo) e as folhas da parte aérea (F) (ciclagem bioquímica) e entre a serapilheira foliar (S) e o folhedo (Fo) (ciclagem biogeoquímica) em três talhões de Eucalyptus grandis no Norte Fluminense

Table 9 - Percent variation of nutrient content between leaf litterfall $(F o)$ and leaf tree $(F)$ (biochemical cycling) and percent variation between leaf litter $(S)$ and leaf litterfall ( $F o$ ) (biogeochemical cycling) in three Eucalyptus grandis stands

\begin{tabular}{|c|c|c|c|c|c|c|c|c|c|c|}
\hline \multirow[t]{2}{*}{ Talhões } & \multicolumn{2}{|c|}{$\mathrm{N}$} & \multicolumn{2}{|c|}{$\mathrm{P}$} & \multicolumn{2}{|c|}{$\mathrm{K}$} & \multicolumn{2}{|c|}{$\mathrm{Ca}$} & \multicolumn{2}{|c|}{$\mathrm{Mg}$} \\
\hline & FoxF $^{1}$ & $\mathrm{SxFo}^{2}$ & FoxF & $\mathrm{SxFo}$ & FoxF & $\mathrm{SxFo}$ & FoxF & SxFo & FoxF & $\mathrm{SxFo}$ \\
\hline & \multicolumn{10}{|c|}{$\%$} \\
\hline $1^{\mathrm{a}}$ rotação & $-46,3$ & $-9,1$ & $-48,0$ & $-15,4$ & $-53,2$ & $-76,7$ & 1,9 & 29,7 & $-21,3$ & $-20,2$ \\
\hline Rebrota 1,5 & $-36,7$ & 2,6 & $-22,0$ & $-16,7$ & $-75,4$ & $-77,9$ & 41,9 & 37,7 & $-29,4$ & $-9,2$ \\
\hline Rebrota 5 & $-48,2$ & 17,0 & $-33,1$ & $-16,2$ & $-78,5$ & $-75,6$ & 25,6 & 10,3 & $-28,4$ & $-24,2$ \\
\hline
\end{tabular}

(1) Folhedo $v s$. folhas $\{([\mathrm{Fo}]-[\mathrm{F}]) /[\mathrm{F}]\} \times 100 \mathrm{e}^{(2)}$ Serapilheira $v s$. folhedo $\{([\mathrm{S}]-[\mathrm{Fo}]) /[\mathrm{Fo}] \times 100$. 
Quadro 10 - Nutrientes no solo $(0-80 \mathrm{~cm})$, na planta e na serapilheira acumulada em talhões de Eucalyptus grandis Table 10 - Soil nutrient $(0-80 \mathrm{~cm})$, in trees and litter in three Eucalyptus grandis stands

\begin{tabular}{|c|c|c|c|c|c|c|c|c|c|c|c|c|}
\hline \multirow[t]{3}{*}{ Talhões } & \multicolumn{4}{|c|}{ Parte aérea } & \multicolumn{4}{|c|}{ Serapilheira } & \multicolumn{4}{|c|}{ Solo } \\
\hline & $\mathrm{P}$ & $\mathrm{K}$ & $\mathrm{Ca}$ & $\mathrm{Mg}$ & $\mathrm{P}$ & $\mathrm{K}$ & $\mathrm{Ca}$ & $\mathrm{Mg}$ & $\mathrm{P}$ & $\mathrm{K}$ & $\mathrm{Ca}$ & $\mathrm{Mg}$ \\
\hline & \multicolumn{12}{|c|}{$\mathrm{kg} \mathrm{ha}^{-1}$} \\
\hline $1^{\mathrm{a}}$ rotação & 13,3 & 258 & 516 & 124,3 & 5,40 & 10,43 & 139 & 30 & 13,9 & 1079,7 & 872,2 & 320,4 \\
\hline Rebrota 1,5 & 5,1 & 105 & 125 & 36,8 & 2,40 & 4,90 & 110 & 17 & 19,2 & 993,8 & 2110,9 & 550,6 \\
\hline Rebrota 5 & 7,9 & 156 & 192 & 71,5 & 3,10 & 4,18 & 88 & 13 & 21,8 & 823,2 & 1385,4 & 384,4 \\
\hline
\end{tabular}

\section{CONCLUSÕES}

1. A sazonalidade da produção de serapilheira foi semelhante nos três talhões, mas a produção total foi superior no talhão em primeira rotação, condicionando, portanto, maior retorno de nutrientes ao solo.

2. As maiores e menores intensidades de ciclagem bioquímica e biogeoquímica foram do $\mathrm{K}$ e $\mathrm{Ca}$, respectivamente.

3. O balanço de nutrientes foi positivo em todos os talhões avaliados, evidenciando-se maior reserva de nutrientes no solo do que absorvido pelas plantas, o que possibilita produtividade similar em rotações futuras.

\section{AGRADECIMENTO}

À Fundação de Amparo à Pesquisa do Estado do Rio de Janeiro (FAPERJ), pelo auxílio financeiro para a realização deste trabalho.

\section{REFERÊNCIAS BIBLIOGRÁFICAS}

ATTIWILL, P.M.; ADAMS, M.A. Nutrient cycling in forests. New Phytology, v. 124, p. 561-582, 1993.

ATTIWILL, P.M.; GUTHRIE, H.B.; LEUNING, R. Nutrient cycling in an Eucalypthus obliqua (L' Herit) forest. I. Litter production and nutrient return. Australian Journal Botanic, v. 26, p. 79-91, 1978.

BARROS, N.F.; NOVAIS, R.F.; NEVES, J.C.L. Fertilização e correção do solo para o plantio de eucalipto. pp 99-186. In: BARROS, N.F. e NOVAIS, R.F. (Eds.). Relação solo-eucalipto. Viçosa, MG: Folha de Viçosa, 1990. 330p.

BIRK,E.M.; SIMPSON, R.W. Steady state and continous input model of litter accumulation and decomposition in Australian eucalypt forests. Ecology, v.6, p. 481-485, 1980.
BRAGA, J.M.; DEFELIPO, B.V. Determinação espectrofotométrica de fósforo em extrato de solo e material vegetal. Revista Ceres, v. 21, p.73-85, 1974.

CALDEIRA, M.V.W. et al. Ciclagem de nutrientes em Acacia mearnsii de Willd.V. quantificação do conteúdo de nutrientes na biomassa aérea de Acacia mearnsii de Willd.V. procedência australiana. Ciência Rural, v.30, p. 977-982, 2000.

DEFELIPO, B.V.; RIBEIRO, A.C. Análise química do solo: metodologia. Viçosa, Minas Gerais, UFV, 1981. 71p. (Boletim de Extensão).

EMPRESA BRASILEIRA DE PESQUISA AGROPECUÁRIA- EMBRAPA - Centro Nacional de Pesquisa de Solos. Rio de Janeiro, Manual de métodos de análise de solo. 2. ed. Rio de Janeiro: 1997. 212p.

FARIA, G. E. et al. Produção e estado nutricional de povoamentos de Eucalyptus grandis, em segunda rotação, em resposta a adubação potássica. Revista Árvore, v. 26, p. 577-584, 2002.

GAMA-RODRIGUES, A.C.; BARROS, N.F.

Ciclagem de nutrientes em floresta natural e em plantios de eucalipto e de dandá no sudeste da Bahia, Brasil. Revista Árvore, v. 26 n.2, p. 193-207, 2002.

GUO, L.B.; SIMS, R.E.H. Litter production and nutrient return in New Zealand eucalypt shortrotation forest: implication for land management. Agriculture Ecossistems and Environment, v. 73, p. 93-100, 1999.

LEITE, F.P. et al. Acúmulo e distribuição de nutrientes em Eucalyptus grandis sob diferentes densidades populacionais. Revista Brasieleira de Ciencia do Solo, v. 22, p. 419-426, 1998. 
LIMA, W. P. Impacto ambiental do

Eucalipto. 2. ed. Piracicaba: EDUSP,1996. 301p.

LISANEWORK, N.; MICHELSEN, A. Litterfall and nutrient release by decomposition in three plantations compared with a natural forest in the Ethiopian highland. Forestry Ecology Management, v. 65 p. 149-164, 1994.

LUIZÃO, F.J.; SCHUBART, H.O.R. Litter production and decomposition in a terra-firme forest of Central Amazonia . Experientia, v.43, p.259-265, 1987.

MORAIS, E.J. et al. Biomassa e eficiência nutricional de espécies de eucalipto em duas regiões bioclimáticas de Minas Gerais. Revista Brasileira de Ciencia do Solo, n.3, p.353-362, 1990.

O'CONNEL, A.M. Nutrient accumulation in and release from the litter layer of karri (Eucalyptus diversicolor) forests of Southwestern Australia. Forestry Ecology Management, v.26, p.95-111, 1989.

REIS, M.G.F.; BARROS, N.F. Ciclagem de Nutrientes em Plantios de Eucalipto. In: BARROS, N.F.; NOVAIS, R.F. (Eds.). Relação solo-eucalipto. Viçosa: Folha de Viçosa, 1990. p. 265-302.
SANTANA, R.C.; BARROS, N.F.; NEVES, J.C.L. Biomassa e conteúdo de nutrientes de procedências de Eucalyptus grandis e Eucalyptus saligna em alguns sítios florestais do Estado de São Paulo. Scientia Florestalis, v. 56, p. 155-169, 1999.

SCHUMACHER, M.V. Estudo da biomassa e dos nutrientes de um povoamento de Eucalyptus globulus (Labillardière) subespécie bicostata. Revista Árvore, v. 22, p. 281-286, 1998.

SCHUMACHER, M.V.; POGGIANI, F.; SIMÕES, J.W. Transferência de nutrientes das copas para o solo através da deposição de folhedo em povoamentos de Eucalyptus camaldulensis, Eucalyptus grandis, Eucalyptus torelliana plantados em Anhembi-SP. IPEF, n. 47, p. 56-61, 1994.

SPANGENBERG, A. et al. Nutrient store and export rates of Eucalyptus urograndis plantation in eastern Amazonia (Jari). Forestry Ecology Management, v. 80, p. 225-234, 1996.

SWIFT, M.J.; HEAL, O.W.; ANDERSON, J.M.

Decomposition in terrestrial ecosystems. Oxford: Blackwell, 1979. 372p.

TEDESCO, M.J.; VOLKWEISS, S.J.; BOHNEN, H. Análises de solo, plantas e outros materiais. Porto Alegre: Universidade Federal do Rio Grande do Sul, 1985. 50p. (Boletim Técnico, 5). 